\title{
THE EFFECTS OF MAGNETIC FIELDS ON THE PROPERTIES OF FERROFLUIDS AND FERROGELS
}

\author{
Ph.J. Camp \\ School of Chemistry, The University of Edinburgh \\ West Mains Road, Edinburgh EH9 3JJ, United Kingdom
}

\begin{abstract}
The effects of magnetic fields on the properties of ferrofluids and ferrogels are examined using computer simulations. Simple microscopic models capture the essential characteristics of magnetic nanoparticles and the suspending medium (fluid or gel). Monte Carlo and molecular dynamics simulations of these models yield macroscopic properties in good agreement with experiment and simultaneously offer unique insights on the microscopic organisation of the particles. Two illustrations are given: the determination of particlesize polydispersity in a ferrofluid using the magnetisation curve, and the mechanical anisotropies of ferrogels induced by uniform magnetic fields.
\end{abstract}

Introduction. There are various ways in which magnetic materials respond to uniform applied magnetic fields. One of the most fundamental examples is the magnetisation curve $M(H)$. Statistical mechanical theories and computer simulations are required to describe this property correctly and accurately, because it depends on the nature and degree of the microscopic correlations between the magnetic dipole moments residing on the constituent particles [1]. An example of the complementarity between theory, experiment and computer simulation in understanding the magnetisation curves of polydisperse colloidal ferrofluids will be discussed in Sec. $2[2,3]$. More subtle responses are observed when a ferrogel is placed in a uniform magnetic field. Some properties - such as the deformation of a finite sample - depend not only on the microscopic correlations between dipole moments, but also on the geometry of the sample. Specifically, the fact that the sample is of macroscopic but finite size requires consideration of demagnetisation fields arising from a non-uniform magnetisation. Typically, particle-based simulations employ periodic boundary conditions (PBCs) to eliminate finite-size effects and to mimic a uniform system in the thermodynamic limit. In general, fixed boundary conditions are of limited use because the numbers of particles which can be handled using current computational resources are insufficient to accommodate domains and magnetisation textures of physically relevant dimensions. These restrictions are discussed in Sec. 1. Other properties, though, can be understood using particle-based methods. Examples include the elastic constants and the magnetisation curve of a ferrogel in the thermodynamic limit, where the presence of boundaries can be neglected. The application of computer-simulation methods to ferrogels will be illustrated in Sec. 3 [4].

1. Computer simulations of magnetic materials. 'Molecular' simulations - such as Monte Carlo (MC) and molecular dynamics (MD) methods almost always rely on the application of PBCs to remove extreme finite-size effects arising from the portion of particles near the edge of the simulation cell [5]. The simulation cell represents a microscopic region of a bulk sample: in reality, it is surrounded by similar but not identical regions; in the simulation, it is sur- 


\section{Ph.J. Camp}

rounded by periodically repeating images. Now, for a finite material - such as a sample of ferrogel or a ferrofluid droplet - there will be a demagnetising field that depends on the sample size and geometry [6]. This field is a crucial factor when considering the macroscopic deformation of a magnetised body. Unfortunately, this is not something that can be captured in a molecular simulation because the non-uniformity of the magnetisation in a finite body occurs on length scales far greater than those that can be represented in a simulation; within PBCs, there is no way of representing a local magnetisation that varies throughout the sample over physically relevant distances. As a result, we are limited to considering a uniform sample with a shape-independent free energy [7]. This is achieved in simulations by evaluating the long-range dipolar interactions between all particles (including the periodic images) using a technique such as Ewald summations with the so-called 'tin-foil' boundary conditions. These boundary conditions cancel out surface poles and remove demagnetisation fields. (Note that in theoretical approaches, it is convenient to consider a prolate ellipsoidal sample of infinite elongation, for which the demagnetisation factor is always zero.) As a result, in what follows, the magnetic potential energy of the system is simply

$$
U_{\text {mag }}=\frac{\mu_{0}}{4 \pi} \sum_{i<j}\left[\frac{\left(\boldsymbol{\mu}_{i} \cdot \boldsymbol{\mu}_{j}\right)}{r_{i j}^{3}}-\frac{3\left(\boldsymbol{\mu}_{i} \cdot \mathbf{r}_{i j}\right)\left(\boldsymbol{\mu}_{j} \cdot \mathbf{r}_{i j}\right)}{r_{i j}^{5}}\right]-\mu_{0} \sum_{i} \mathbf{H} \cdot \boldsymbol{\mu}_{i},
$$

where $\boldsymbol{\mu}_{i}$ is the dipole moment on particle $i, \mathbf{r}_{i j}$ is the separation vector between particles $i$ and $j, r_{i j}=\left|\mathbf{r}_{i j}\right|$, and $\mathbf{H}$ is an applied magnetic field. In MD simulations, classical equations of motion are integrated numerically. In MC simulations (used here), configurations of an $N$-particle system are sampled with the Boltzmann probability proportional to $\exp \left(-U / k_{\mathrm{B}} T\right)$, where $U$ is the total potential energy which includes $U_{\text {mag }}$, short-range interactions (here, hard-sphere interactions $U_{\mathrm{HS}}$ ), and any additional contributions.

2. Magnetisation curves of ferrofluids. The magnetisation curve $M(H)$ of a real colloidal ferrofluid depends on the temperature, the concentration, and the particle-size polydispersity (mainly through the distribution of particle dipole moments). If a reliable theoretical prediction for $M(H)$ with an arbitrary particle-size distribution is available, then this distribution can be optimised against experimental results to gain information on the composition of the ferrofluid. 'Reliable' here means that the theory gives a consistent and effectively complete description of dipolar orientational correlations over the appropriate ranges of concentration and temperature. This is best assessed against computer simulation data, because then the particle-size distribution can be prescribed in both the theory and the simulation, and the effects of concentration and temperature can be isolated. As an example, consider the magnetisation curves presented in Fig. 1 [2, 3]. The experimental points are from a magnetite ferrofluid synthesised by the Perm group [8], measured at $T=293 \mathrm{~K}$. The different data sets correspond to successive dilutions of a ferrofluid with high concentration characterised by the saturation magnetisation $M_{\mathrm{s}}=\varphi m_{\mathrm{s}}$ indicated in the figure; here, $\varphi$ is the magnetic volume fraction and $m_{\mathrm{s}}=480 \mathrm{kA} / \mathrm{m}$ is the saturation magnetisation of magnetite. Note that the particle-size distribution must be the same in each case. The curves are fitted using Ivanov and Kuznetsova's second-order modified mean-field theory (MMFT2) for dipolar hard spheres with the distribution given by

$$
p(\sigma)=\frac{\sigma^{\alpha} \exp \left(-\sigma / \sigma_{0}\right)}{\sigma_{0}^{\alpha+1} \Gamma(\alpha+1)},
$$




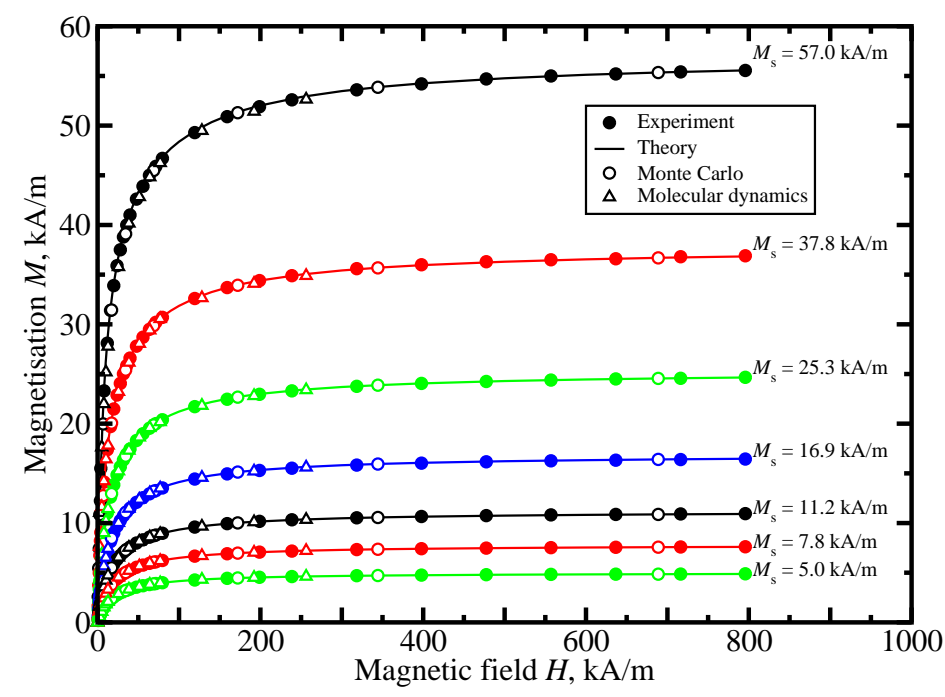

Fig. 1. Magnetisation curves of ferrofluids from experiments (solid points), MMFT2 (lines), MC simulations (open circles), and MD simulations (open triangles). $M_{\mathrm{s}}=\varphi m_{\mathrm{s}}$ is the saturation magnetisation for a fluid with magnetic volume fraction $\varphi$ and magnetite saturation magnetisation $m_{\mathrm{s}}=480 \mathrm{kA} / \mathrm{m}$.

where $\sigma$ is the magnetic-core diameter, and $\sigma_{0}=1.2266 \mathrm{~nm}$ and $\alpha=4.9518$ are the fitted parameters. Several alternative theories were tested in Ref. [2], but MMFT2 was the only approach that gave consistent results for $p(\sigma)$ across the whole concentration range. Of course, you can fit any data with a sufficiently complicated function, so to check for consistency, computer simulations were used to calculate $M(H)$ for a fluid of dipolar hard spheres with the fitted $p(\sigma)$; no approximations are made in the simulations and the results are essentially exact for a given $p(\sigma)$. Fig. 1 demonstrates complete agreement between experiment, theory and computer simulation, showing that the theory is indeed reliable.

3. Ferrogels in uniform magnetic fields. Zrínyi and co-workers have measured the responses of ferrogels in uniform magnetic fields. Moreover, they have explored the effect of applying fields during the synthesis of ferrogels; the fields enhance the positional correlations between colloidal magnetic particles, which are then locked in to the polymeric gel matrix by cross-linking reactions. If $\mathbf{H}_{\mathrm{f}}$ denotes the field during synthesis, and $\mathbf{H}_{\mathrm{g}}=\left(H_{x}, 0,0\right)$ denotes the field applied to the resulting ferrogel, then three situations are possible: (a) $\mathbf{H}_{\mathrm{f}}=0$, (b) $\mathbf{H}_{\mathrm{g}} \| \mathbf{H}_{\mathrm{f}}=(H, 0,0)$ and (c) $\mathbf{H}_{\mathrm{g}} \perp \mathbf{H}_{\mathrm{f}}=(0,0, H)$. In experiments, it is observed that an applied field leads to a large increase in the elastic modulus in the field direction, and smaller increases in the perpendicular directions, and that the greatest enhancement is obtained when $\mathbf{H}_{\mathrm{g}} \| \mathbf{H}_{\mathrm{f}}$ [9]. Filipcsei and Zrínyi have explored the swelling of ferrogels in uniform magnetic fields and have proposed a thermodynamic model to explain the observations [10]. As explained in Sec. 1, the deformation of a macroscopic sample cannot be handled using molecular-simulation methods, but the elastic properties are definitely within reach [4]. The microscopic model studied here is constructed from fluid-phase configurations of dipolar hard 
spheres generated from MC simulations and subsequently trapped in an elastic matrix. The potential energy is augmented by an elastic term to give

$$
U=U_{\mathrm{HS}}+U_{\mathrm{mag}}+\frac{1}{2} G_{0} L_{0}^{3} \sum_{\alpha=x, y, z}\left(\frac{L_{\alpha}}{L_{0}}-1\right)^{2},
$$

where $G_{0}$ and $L_{0}$ are the elastic modulus and equilibrium length, respectively, of a cubic sample of pure gel. MC simulations of the resulting ferrogel are performed by Boltzmann sampling the orientations of the particle dipole moments and each of the box lengths $L_{\alpha}$ independently. The particles are assumed to be locked in to the elastic matrix, and so a key approximation is that the particle configurations undergo affine transformations as the box lengths fluctuate. The elastic modulus in each direction is measured using the fluctuation formula

$$
G_{\alpha}=\frac{k_{\mathrm{B}} T}{V\left\langle\epsilon_{\alpha}^{2}\right\rangle}
$$

where $\epsilon_{\alpha}=L_{\alpha} /\left\langle L_{\alpha}\right\rangle-1$ is the instantaneous strain. Fig. 2 shows $G_{\alpha}$ as a function of magnetic field $H_{x}$. The simulations were carried out in reduced units, but for illustrative purposes, the data in Fig. 2 are shown as for a ferrogel containing
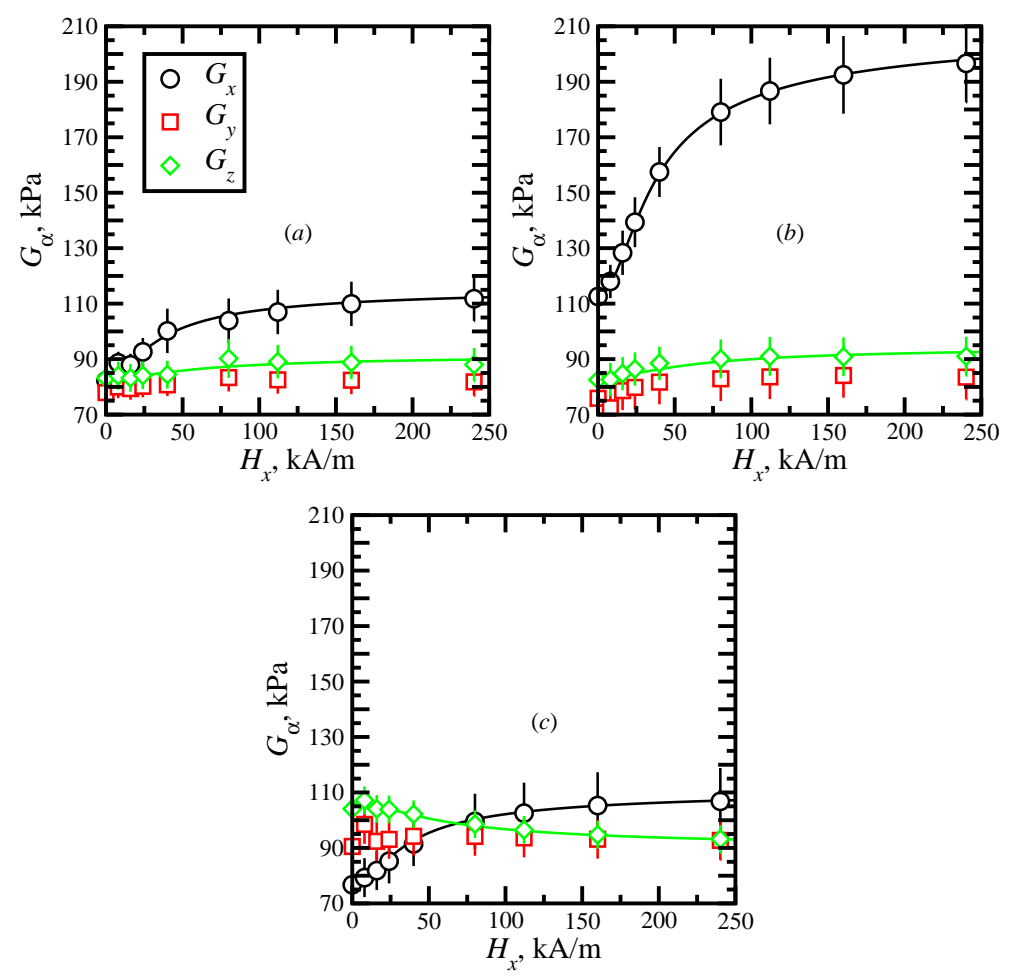

Fig. 2. Elastic moduli of a model ferrogel with magnetic volume fraction $\varphi \simeq 0.1$ as functions of the applied magnetic field $\boldsymbol{H}_{\mathrm{g}}=\left(H_{x}, 0,0\right):(a)$ ferrogel prepared with $\boldsymbol{H}_{\mathrm{f}}=\mathbf{0} ;(b)$ ferrogel prepared with $\boldsymbol{H}_{\mathrm{f}}=(80,0,0) \mathrm{kA} / \mathrm{m} ;(c)$ ferrogel prepared with $\boldsymbol{H}_{\mathrm{f}}=(0,0,80) \mathrm{kA} / \mathrm{m}$. Points are from simulations and the lines are fits. Data are given for $10 \mathrm{~nm}$ magnetite particles at $T=293 \mathrm{~K}$ dispersed in a gel with elastic modulus $G_{0}=41 \mathrm{kPa}$. 
The effects of magnetic felds on the properties of ferrofluids and ferrogels

$10 \% \mathrm{v} / \mathrm{v}$ magnetite particles of diameter $\sigma=10 \mathrm{~nm}$, at $T=293 \mathrm{~K}$, and with a pure-gel elastic modulus $G_{0}=41 \mathrm{kPa}$ (equal to $\left.10 k_{\mathrm{B}} T / \sigma^{3}\right)$. A magnetic field $\left(\mathbf{H}_{\mathrm{f}}\right.$ ) of $80 \mathrm{kA} / \mathrm{m}$ was applied to the fluid in Figs. $2(b, c)$; this gave a magnetisation of about 0.8 times the saturation value. Fig. $2(b)$ shows that when $\mathbf{H}_{\mathrm{f}}$ is parallel to $\mathbf{H}_{\mathrm{g}}$, the elastic modulus in the field direction is enhanced by almost $100 \%$, whereas the enhancement in the other cases is significantly less. This is in good agreement with experiments. The microscopic origin of this effect is the presence of chain-like positional correlations induced by $\mathbf{H}_{\mathrm{f}}$ [4], which in turn leads to an increase in the curvature of the energy (consisting of magnetic and elastic terms). One pathology of the model is evident in the dependence of $G_{z}$ on $H_{x}$ shown in Fig. 2(c); the elastic modulus decreases with increasing magnetic field, due to side-by-side parallel repulsions between dipoles in chains 'softening' the magnetic interactions. This arises from the restriction of affine transformations; buckling and other non-affine transformations should be built in to the model, but this will lead to technical complications. A more straightforward approach is to model the polymer matrix explicitly, albeit with an increase in computational cost. Finally, it was found heuristically that $G_{x}\left(H_{x}\right)$ and $G_{z}\left(H_{x}\right)$ depend linearly on $M_{x}^{2}\left(H_{x}\right)$ and $M_{x}^{4}\left(H_{x}\right)$, respectively, and that the MMFT2 theory provides an excellent description of the ferrogel magnetisation curves [4]. Combining these relations leads to the curves shown in Fig. 2. A theory of the magnetisation dependences of $G_{\alpha}$ has not yet been found.

4. Conclusion. It has been shown that 'molecular' simulation techniques can be applied to study the responses of ferrofluids and ferrogels to uniform magnetic fields. The limitations of such microscopic techniques are acknowledged; MC and MD simulations are not magic bullets. Nonetheless, some aspects of magnetic response can be studied in detail. In the examples summarised here, simulations can be used to test rigorously theoretical models of the magnetisation curves of ferrofluids, and to yield insight on the microscopic mechanisms leading to fielddependent elastic enhancement and anisotropy in ferrogels.

Acknowledgements. It is a pleasure to acknowledge the contributions to this work by Dean Wood (Edinburgh), Alexey Ivanov and Sofia Kantorovich (Ekaterinburg), and Christian Holm (Stuttgart).

\section{REFERENCES}

[1] A.O. Ivanov and O.B. Kuznetsova. Magnetic properties of dense ferrofluids: An influence of interparticle correlations. Phys. Rev. E, vol. 64 (2001), p. 041405 .

[2] A.O. Ivanov, et al. Magnetic properties of polydisperse ferrofluids: A critical comparison between experiment, theory, and computer simulation. Phys. Rev. E, vol. 75 (2007), p. 061405.

[3] A.O. Ivanov, et al. Magnetic measurements as a key for the particle size distribution in ferrofluids: experiment, theory, and computer simulations. Magnetohydrodynamics, vol. 43 (2007), pp. 393-400.

[4] D.S. Wood And P.J. CAmP. Modeling the properties of ferrogels in uniform magnetic fields. Phys. Rev. E, vol. 83 (2011), p. 011402.

[5] D. Frenkel and B. Smit. Understanding Molecular Simulation: From Algorithms to Applications (Academic Press, San Diego, 2001), 2nd ed. 
[6] L.D. Landau And E.M. Lifshitz. Electrodynamics of Continuous Media (Pergamon Press, Oxford, 1960), 1st ed.

[7] S. Banerjee, R.B. Griffiths, And M. Widom. Thermodynamic Limit for Dipolar Media. J. Stat. Phys., vol. 93 (1998), pp. 109-141.

[8] A.F. Pshenichnikov, V.V. Mekhonoshin, and A.V. Lebedev. Magnetogranulometric analysis of concentrated ferrocolloids. J. Mag. Mag. Mater., vol. 161 (1996), pp. 94-102.

[9] Z. Varga, G. Filipcsei, And M. Zrínyi. Smart composites with controlled anisotropy. Polymer, vol. 46 (2005), pp. 7779-7787.

[10] G. FilipCsei AND M. ZRÍNYi. Magnetodeformation effects and the swelling of ferrogels in a uniform magnetic field. J. Phys.: Condens. Matter, vol. 22 (2010), p. 276001.

Received 03.06.2011 